Case Report

\title{
Unusual anatomy of a maxillary first molar with two palatal roots: a case report
}

\author{
Flávia S. F. Tomazinho ${ }^{1)}$, Flares Baratto-Filho ${ }^{1,2)}$, Suellen Zaitter ${ }^{1)}$, \\ Denise P. Leonardi ${ }^{1)}$ and Carla C. Gonzaga ${ }^{1)}$ \\ ${ }^{1)}$ Department of Dentistry, Positivo University, Curitiba, PR, Brazil \\ ${ }^{2)}$ Department of Dentistry, University of Joinville, Joinville, SC, Brazil
}

(Received 27 April and accepted 21 October 2009)

\begin{abstract}
The success of endodontic therapy is based on good endodontic access, correct cleaning and shaping, and adequate root canal obturation. However, endodontic treatment is also dependent on a sound knowledge of the internal anatomy of human teeth, especially when anatomical variations are present. Certain anatomical changes may be present to varying degrees, and it is important to report these to improve the understanding and expertise of endodontic specialists. With this aim in mind, this study reports an example of a maxillary first molar showing unusual anatomy (four roots and six root canals) and describes the endodontic treatment that was employed. (J Oral Sci 52, 149-153, 2010)
\end{abstract}

Keywords: anatomical variations; maxillary molars; root canal.

\section{Introduction}

The success of endodontic therapy is strictly dependent on the accomplishment of all treatment steps, especially the complete removal of bacteria and bacterial products from the root canal system during the cleaning and shaping procedure. Variations in dental anatomy are found in all groups of teeth, and a knowledge of these variations, particularly in relation to the location and treatment of all canals, is the key to successful endodontic therapy, since

Correspondence to Dr. Flares Baratto-Filho, Rua Geraldo Lipka 65, Ap. 101, 81200-590, Curitiba, PR, Brazil

Tel: +55-41-33173406

Fax: +55-41-3317-3082

E-mail: fbaratto@uol.com.br \& flares@up.edu.br the ability to find and properly treat all root canals may prevent future failures (1). Maxillary first molars are particularly noteworthy for anatomical variations of the root canals (2). Therefore, in order to achieve successful clinical results, a complete clinical and radiographic examination and a thorough knowledge of the morphology of these teeth are necessary (3).

Regarding the internal anatomy of maxillary molars, special attention should be paid to the mesiobuccal root, where the fourth canal (MB2) is frequently located (1-5). Baratto-Filho et al. (2) reported that, in first maxillary molars, the frequency of extra roots and canals in the mesiobuccal root (MB2) was $92.85 \%$ (based on ex-vivo results), $95.63 \%$ (clinical results) and $95.45 \%$ (cone beam computed tomography - CBCT - results), whereas the corresponding figures for the distobuccal root (DB2) were $5.10 \%$ (ex-vivo results) and $3.75 \%$ (clinical results), and those for the palatal root were $2.05 \%$ (ex-vivo results), $0.62 \%$ (clinical results) and $4.55 \%$ (CBCT results). Pécora et al. (5) investigated the internal anatomy of 370 cleared maxillary molars and observed that the first, second and third molars presented three canals in $75 \%, 58 \%$ and $68 \%$ of cases, respectively, whereas four canals were present in $25 \%, 42 \%$ and $32 \%$, respectively; these fourth canals were located in the mesiobuccal root in all cases. Although these findings are informative, the number of roots and canals may vary, and it is very important to consider the populations investigated, as well as the number of teeth included in each study. One study investigated the presence of significant anatomical variations in 338 maxillary first molars (6), and reported three cases of teeth with six root canals, comprising three in the mesiobuccal root, two in the distobuccal root, and one in the palatal root. 
The occurrence of two palatal roots should also be mentioned, since this anatomical variation is frequently reported. One example is a study that demonstrated some variations in the palatal root of maxillary molars, including a single root with two openings of separate canals, two separate canals and two independent foramina; two separate roots, each with one root canal opening, one root canal and one foramen; and a third situation with a single root with one root canal opening, one bifurcated root canal and two foramina (7).

When the mesiobuccal root had a single canal, the internal root canal system morphology reflected the external root anatomy. In fact, the canal was oval in the coronal and middle third, having a tendency to be more circular in the apical portion of the root. However, it is important to note that when a second mesiobuccal root canal was present, its localization and negotiation were reported to be difficult in $20.8 \%$ of cases, due to its configuration (8).

On the basis of the aforementioned literature, this paper reports an example of a maxillary first molar with an unusual anatomy (four roots and six canals) and describes the importance and difficulties of the endodontic treatment,
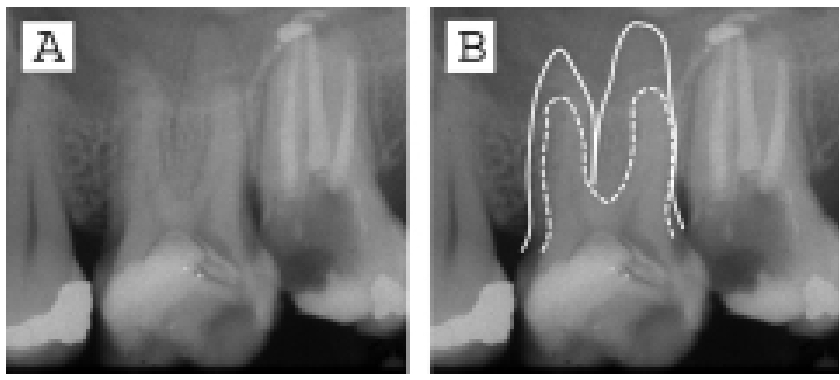

Fig. 1 (A) Initial radiograph of the maxillary left first molar, orthoradial projection; (B) The full line indicates the palatal root and the dashed line indicates the buccal root. since the localization of root canals is considered a challenge in dental practice.

\section{Case Report}

A 32-year-old male presented at the university clinic upon referral from a dental practitioner for endodontic treatment of the maxillary left first molar. The pulp chamber had already been assessed and the patient did not present any symptomatology. On this basis, pulp necrosis was diagnosed, and this was confirmed by thermal pulp testing (Endo Frost, Roeko, Langenau, Germany). Percussion and palpation tests were negative, and no systemic complications were reported in the patient's medical history. Radiographic examination (Fig. 1) revealed the presence of four roots, two buccal and two palatal.

After confirmation of the diagnosis, anesthesia was performed, followed by placement of a rubber dam and coronal opening, which revealed the presence of six root canals during negotiation of the four roots, arranged as follows:

- two canals in the distobuccal root (DB) (ovoid root): DB1 and DB2 (Fig. 2);

- two canals in the mesiobuccal root (MB) (ovoid root): MB1 and MB2 (Fig. 3);

- one canal in the mesiopalatal root: MP (Fig. 4);

- one canal in the distopalatal root: DP (Fig. 4).

All root canals are shown in Fig. 5. After negotiation of the six canals, they were prepared using the crown-down technique with ProFile 0.04 instruments (DentsplyMaillefer, Ballaigues, Switzerland) and irrigated with $2.5 \%$ sodium hypochlorite. Apical preparation was performed with \#40 instruments for all roots, and confirmed with manual instruments. Final irrigation was performed with 17\% EDTA followed by root canal drying and placement of a calcium hydroxide root canal dressing (Calen, SS White, Rio de Janeiro, Brazil).


Fig. 2 Assessment of DB1 and DB2 openings with K files n. 10. 
After 15 days, the patient returned for obturation. Anesthesia and placement of a rubber dam were performed again, and the calcium hydroxide dressing was removed with aid of a manual file under irrigation with $2.5 \%$ sodium hypochlorite. The root canals were then obturated with gutta-percha points (Tanari, Manacapuru, AM, Brazil) and an endodontic sealer, AH-Plus (Densply-Maillefer) thermally softened with a gutta-percha condenser (DensplyMaillefer). Final radiographs were taken in orthoradial (Fig. 6A) and distoradial (Fig. 6B) projections to assess the

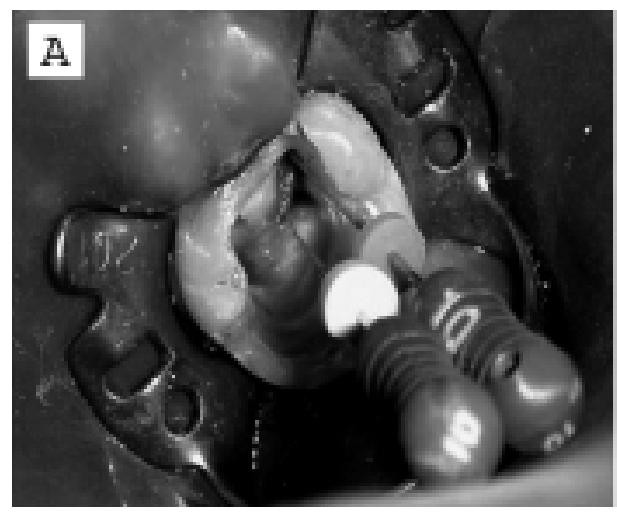

Fig. 3 Assessment of MB1 and MB2 openings with K files n. 10.

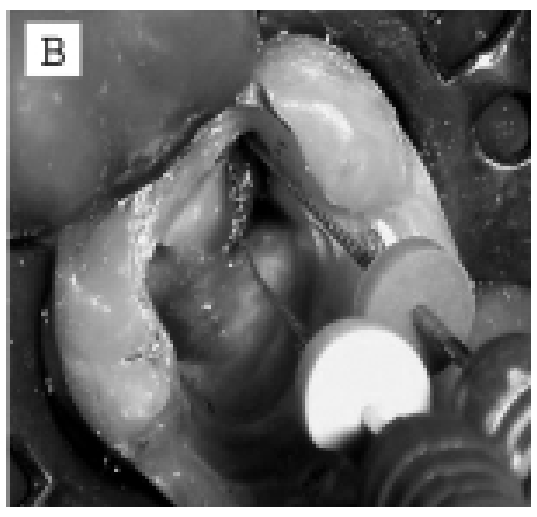

Fig. 4 Occlusal view of openings on the mesiobuccal (MB), distobuccal (DB), mesiopalatal (MP) and distopalatal (DP) roots.
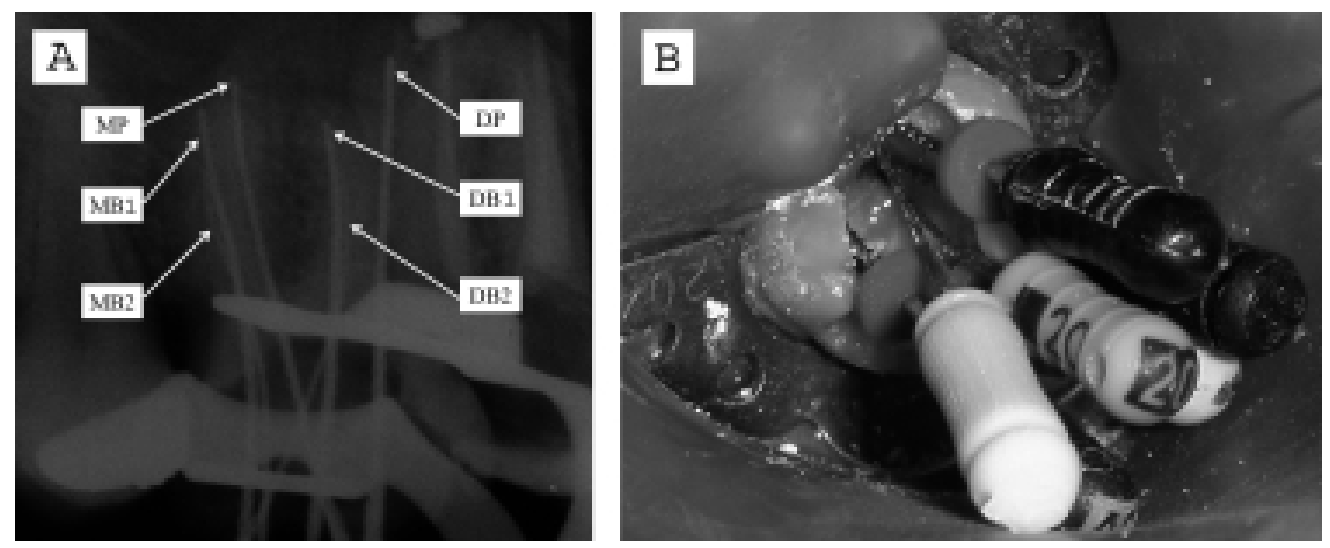

Fig. 5 (A) Radiograph demonstrating the six root canals; (B) The six files used for measurement of root canals.
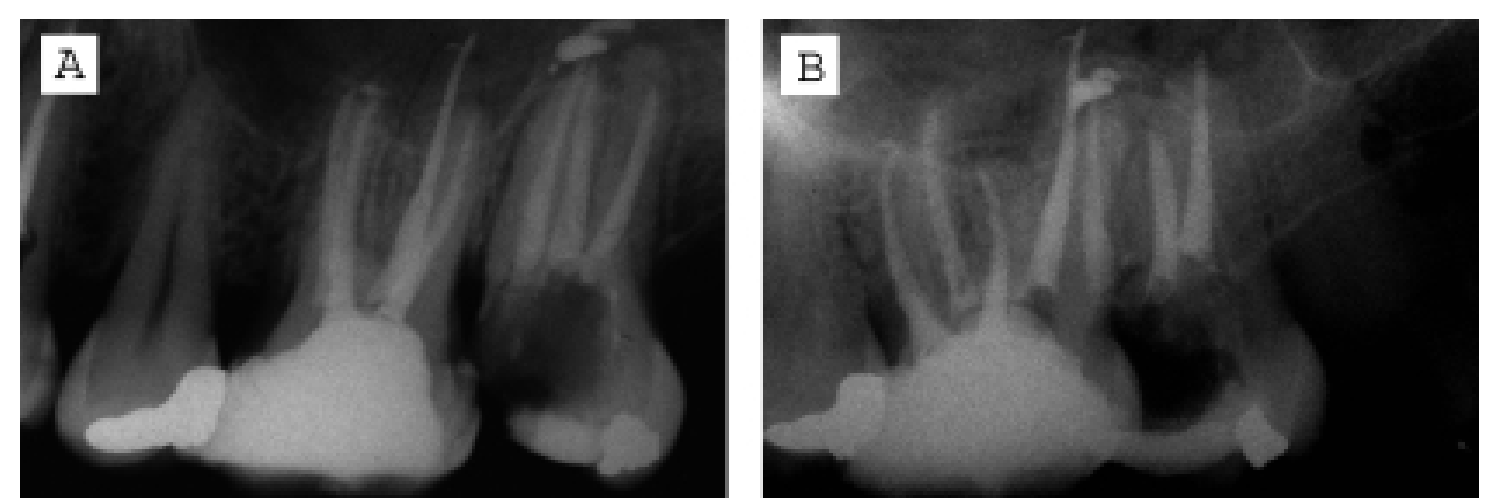

Fig. 6 Final radiograph of the obturation (orthoradial (A) and distoradial projection (B)). 
quality of final obturation, which revealed an overflow at the distopalatal and mesiobuccal roots. Because the roots were oval, the extra canals in the buccal roots became fused after cleaning and shaping, and were not evident in the final radiographs.

The patient was scheduled for follow-up appointments after 15 days, and then referred for final restoration.

\section{Discussion}

For successful endodontic therapy, dentists should devote equal care to all steps of the endodontic treatment. However, one of the greatest concerns of endodontists is the cleaning, shaping and disinfection of the root canal. The internal anatomy of human teeth is complex and variable. A tooth does not present just a simple root canal, but a complex system of root canals composed of lateral, collateral, recurrent, secondary, accessory and multiple foraminal openings, whose cross-sections can be circular or oval. Therefore, the cleaning of root canals is a challenge. According to Clark (9), the root canal system can be ovoid in some cases, making the location, cleaning and obturation of extra canals more difficult, since rotary instruments and obturation points of gutta-percha are round, due to mechanical limitations. This situation means that their shapes are anatomically appropriate for round roots, but not for ovoid roots.

Weine (10) indicated that the root canal system can be classified into four types: type I is a single canal from the pulp chamber to the apex; type II describes two separate canals near the pulp chamber but converging to form a single canal near the apex; type III describes two separate canals emerging and ending in distinct apical foramina; type IV is related to one canal emerging from the pulp chamber and dividing near the apex in two separate canals with separate foramina. According to the same author, type II is the most frequent, being present in more than 55\% of cases. Another widely used classification is the one proposed by Vertucci (11), where the variations in the number of canals present in a single root can be divided into: type I, single canal; type II, two separate canals that converge near the apex; type III, a canal that is divided in two within the root, converging to a single canal near the apex; type IV, two separate canals from the pulp chamber to the apex; type $\mathrm{V}$, a canal that is divided in two before the apex; type VI, two canals that converge within the root and are divided into two separate canals before the apex; type VII, a canal that is divided, then converges within the root and is again divided in two at the apex; type VIII, three separate canals extending from the pulp chamber to the apex. According to these classifications $(10,11)$, the case described in the present report was type II (mesiobuccal and distobuccal roots) and type I (mesiopalatal and distpalatal roots).

One notable study describing maxillary molars with two palatal roots was that of Christie et al. (12), who suggested a classification for such teeth based on the degree of separation of their roots and their divergence: Type I maxillary molars having two widely divergent palatal roots that are often long and tortuous, the buccal roots often being "cow-horn" shaped and less divergent. Radiographically, this type shows four separate root apices. Type II maxillary molars having four separate roots, although the roots are often shorter, run parallel, have a buccal and lingual root morphology, with blunt root apices. Radiographically, buccolingual superimposition may give an impression that only one mesial and distal root is present. Type III maxillary molars with a constricted root morphology, the mesiobuccal, mesiopalatal, and distopalatal canal being encaged in a web of root dentin. The distobuccal root in such cases appears to stand alone, and may even diverge distobuccally. Based on this classification, Di Fiore (13) reported a case of a maxillary first molar with four roots, classified as type II. Similarly, Baratto-Filho et al. (14) reported three maxillary molars with two palatal roots (one case report and two cleared teeth), comprising two type I molars and one molar that could not be accommodated by the Christie et al. (12) classification, thus being suggested as type IV. The maxillary molar in the present case may have been classified as type I, as it demonstrated completely independent roots and canals. Another important study was conducted by Peikoff et al. (15), who indicated that the frequency of this variation of two palatal roots was nearly $1.4 \%$. That study (15) also supported the results of Görduysus et al. (16) in terms of the distance between the canals in the mesiobuccal root, i.e. between $1 \mathrm{~mm}$ and $2 \mathrm{~mm}$. The same authors claimed that mesiobuccal canals frequently do not terminate in independent foramina, and that their treatment is clinically important. Overlooking the canal may allow untreated microorganisms to colonize the space, leading to infection and treatment failure. Even partial treatment of the canal will improve the chance of success.

With regard to variations in the number of canals, the most common occurrence in maxillary molars is a fourth canal in the mesiobuccal root, since this root tends to present an oval cross-section, as mentioned by Pécora et al. (5) and Baratto-Filho et al. (2). However there may be a larger variation in the number of root canals, as described by Beatty (17), who reported a maxillary first molar with five root canals (three in the mesiobuccal root). Some years later, Bond et al. (18) also reported a maxillary first molar with six root canals, comprising two in the 
mesiobuccal root, two in the distobuccal root, and two in the palatal root. This arrangement of the root canals was in accord with the present case. However Maggiore et al. (19) reported a maxillary first molar with six canals that presented a conformation different from that in the present case, with three root canals in the palatal root, two in the mesiobuccal root, and one in the distobuccal root. Besides the location of the canals, the present case also differed from that reported by Maggiore et al. (19) in the ovoid crosssection of the roots, with two canals in the mesial root and two canals in the distal root, which merged after cleaning and shaping.

Despite the fact that, in this case, the buccal canals were not considered to be independent, their location and negotiation are very important, because according to Holderrieth and Gernhardt (20), the main reasons for endodontic failure are apical percolation and the presence of microorganisms caused by complete instrumentation, inadequate cleaning, insufficient canal obturation, and the presence of untreated canals. Therefore, a thorough knowledge of root and root canal morphology and a good anticipation of their possible morphologic variations are essential, and will help to reduce endodontic failure caused by incomplete root canal preparation and obturation.

Finally, the present case suggests a possible anatomical variation in maxillary first molars, namely the occurrence of four distinct root canals and two root canals due to the presence of a root that is oval in section. Although the presence of six root canals complicates the instrumentation and obturation procedures, it is not an obstacle to successful endodontic therapy.

\section{References}

1. Cobankara FK, Terlemez A, Orucoglu H (2008) Maxillary first molar with an unusual morphology: report of a rare case. Oral Surg Oral Med Oral Pathol Oral Radiol Endod 106, e62-65.

2. Baratto Filho F, Zaitter S, Haragushiku GA, de Campos EA, Abuabara A, Correr GM (2009) Analysis of the internal anatomy of maxillary first molars using different methods. J Endod 35, 337342.

3. Adanir N (2007) An unusual maxillary first molar with four roots and six canals: a case report. Aust Dent J 52, 333-335.

4. Jafarzadeh H, Javidi M, Zarei M (2006) Endodontic retreatment of a maxillary second molar with three separate buccal roots. Aust Endod J 32, 129-132.
5. Pécora JD, Woelfel JB, Sousa Neto MD, Issa EP (1992) Morphologic study of the maxillary molars. Part II: internal anatomy. Braz Dent J 3, 53-57.

6. Martínez-Berná A, Ruiz-Badanelli P (1983) Maxillary first molars with six canals. J Endod 9, 375-381.

7. Stone LH, Stroner WF(1981) Maxillary molars demonstrating more than one palatal root canal. Oral Surg Oral Med Oral Pathol 51, 649-652.

8. Somma F, Leoni D, Plotino G, Grande NM, Plasschaert A (2009) Root canal morphology of the mesiobuccal root of maxillary first molars: a micro-computed tomographic analysis. Int Endod J 42, 165-74.

9. Clark DJ (2005) Shaping and restoring ovoid canal systems. Endodontic Therapy 5, 9-13.

10. Weine FS (1998) Endodontic treatment. 5th ed, Santos, São Paulo, 240-278.

11. Vertucci FJ (1984) Root canal anatomy of the human permanent teeth. Oral Surg Oral Med Oral Pathol, 58, 589-599.

12. Christie WH, Peikoff MD, Fogel HM (1991) Maxillary molars with two palatal roots: a retrospective clinical study. J Endod 17, 80-84.

13. Di Fiore PM (1999) A four-rooted quadrangular maxillary molar. J Endod 25, 695-697.

14. Baratto-Filho F, Fariniuk LF, Ferreira EL, Pécora JD, Cruz-Filho AM, Sousa-Neto MD (2002) Clinical and macroscopic study of maxillary molars with two palatal roots. Int Endod J 35, 796-801.

15. Peikoff MD, Christie WH, Fogel HM (1996) The maxillary second molar: variations in the number of roots and canals. Int Endod J 29, 365-369.

16. Görduysus MÖ, Görduysus M, Friedman S (2001) Operating microscope improves negotiation of second mesiobuccal canals in maxillary molars. $\mathrm{J}$ Endod 27, 683-686.

17. Beatty RG (1984) A five-canal maxillary first molar. J Endod 10, 156-157.

18. Bond JL, Hartwell G, Portell FR (1988) Maxillary first molar with six canals. J Endod 14, 258-260.

19. Maggiore F, Jou YT, Kim S (2002) A six-canal maxillary first molar: case report. Int Endod J 35, 486-491.

20. Holderrieth S, Gernhardt CR (2009) Maxillary molars with morphologic variations of the palatal root canals: a report of four cases. J Endod 35, 1060-1065. 